\title{
Quantitative Differential Phase Contrast Imaging of the Magnetostructural Transition and Current-driven Motion of Domain Walls in FeRh Thin Films
}

\author{
Trevor P. Almeida ${ }^{1}$, Rowan Temple ${ }^{2}$, Jamie Massey ${ }^{2}$, Kayla Fallon ${ }^{1}$, Gary Paterson ${ }^{1}$, Thomas Moore ${ }^{2}$, \\ Damien McGrouther ${ }^{1}$, Christopher H. Marrows ${ }^{2}$ and Stephen McVitie ${ }^{1}$ \\ 1. SUPA, School of Physics and Astronomy, University of Glasgow, Glasgow, UK. \\ ${ }^{2}$ School of Physics and Astronomy, University of Leeds, Leeds, UK.
}

The ordered $\alpha^{\prime}$ alloy of iron-rhodium $\left(\mathrm{Fe}_{48} \mathrm{Rh}_{52}\right.$ to $\left.\mathrm{Fe}_{56} \mathrm{Rh}_{44}\right)$ has attracted considerable attention due to its magnetostructural transition from its antiferromagnetic (AF) to ferromagnetic (FM) phase [1]. $\alpha^{\prime}$-FeRh undergoes a first-order phase transition from its room-temperature AF state to FM between $\sim 350$ to $380 \mathrm{~K}$, and can hence present phase AF/FM co-existence, separated by phase-boundaries (PBs). Controlled nucleation and movement of these PBs is considered very desirable for potential use in magnetic data storage applications [2]. Previous studies have shown that the PBs can be created and driven in FeRh films by combining heating with differential gradients of chemical doping [3]. However, our knowledge of the dynamic behaviour of PBs or domain walls (DWs) between FM regions in FeRh is often limited to bulk magnetic measurements or low magnification imaging, i.e. Kerr microscopy. In order to fully understand the magnetostructural transition and dynamic motion of DWs or PBs, it is necessary to examine the effects of temperature and electrical current pulses (CPs) directly. The scanning transmission electron microscopy (STEM) technique of differential phase contrast (DPC) imaging permits nanometre-scale imaging of magnetisation within nanostructured thin films as a function of applied electric and magnetic fields, as well as temperature. This is the only technique that is presently able to provide both highresolution images of DW motion in thin magnetic films in situ within the TEM and quantitative measurements of saturation induction from the DWs, directly. Here, the first use of DPC to examine the magnetostructural transition in nano-scale FeRh thin films during heating is presented. In addition, the application of CPs allows for the examination of the current-driven motion of DWs during combined in situ heating and biasing.

Thin films of ordered $\alpha^{\prime}$-FeRh alloy were grown epitaxially on clean (001) MgO substrates or NiAl buffer layers by DC magnetron sputter co-deposition, as described previously [4]. Cross-sectional and planar FeRh TEM samples were prepared from bulk substrates and transferred onto in situ heating (DENSsolution Wildfire ${ }^{\mathrm{TM}}$ ) or combined in situ heating / biasing (DENSsolution Lightning ${ }^{\mathrm{TM}} \mathrm{D9}^{+}$) TEM electronic (e-) chips by focused ion beam methods [4]. Additional planar FeRh samples were prepared through HF-etching of the substrates, transferred onto Cu TEM grids and heated up to $100^{\circ} \mathrm{C}$ using a Gatan heating holder. Conventional and high-resolution STEM imaging of the FeRh films and substrates was performed using a JEOL ARM cFEG instrument at 200kV, whilst energy dispersive X-ray (EDX) spectroscopy provided chemical analysis. The magnetic structure of the films is visualised using DPC imaging under low-magnetic field conditions or with an in situ applied field. In addition, in situ heating up to $450^{\circ} \mathrm{C}$ using the DENSsolution e-chips provides direct access to the magnetostructural transition and thermomagnetic behaviour of the DWs within the FeRh films, whilst DW motion is induced through systematic application of CPs ranging from $\sim 5^{*} 10^{9}-5^{*} 10^{10} \mathrm{~A} / \mathrm{m}^{2}$.

Figure 1 presents a cross- and planar-section of the FeRh thin film, providing information on its thickness, chemical distribution and interface with the NiAl buffer and GaAs substrate [5]. The dark-field (DF) STEM image of Fig. 1a reveals the FeRh film to be grown with a thickness of $\sim 50 \mathrm{~nm}$, whilst the EDX chemical maps (Figs 1b) acquired from the boxed region (red) in Fig. 1a displays the elemental distribution of rhodium, iron, nickel, aluminium, arsenic and gallium, respectively. The DPC image of Fig. 1c shows well-resolved DWs between FM regions (arrowed) at $120^{\circ} \mathrm{C}$ and measurements of electron-beam deflection from the DW (boxed region, red) as a function of temperature allowed for calculation of integrated magnetic induction, and subsequent quantitative charting of the magnetostructural transition (Fig. 1d). Hence, the STEM imaging, EDX analysis and quantitative DPC imaging presented in Figure 1 has confirmed the epitaxially-grown FeRh thin film is indeed the ordered $\alpha$ ' phase and exhibits the AF/FM magnetostructural transition. 
The DPC images of planar FeRh thin films in Figure 2 present information on the AF/FM magnetostructural transition as a function of temperature and CP-induced DW motion. Fig. 2a reveals the nucleation of vortex magnetic domains (labelled ' $\mathrm{v}$ ') in the HF-etched planar FeRh sample at $75^{\circ} \mathrm{C}$ and provides evidence for a condition of $\mathrm{AF} / \mathrm{FM}$ phase co-existence. Further heating to $100^{\circ} \mathrm{C}$ (Fig. 2b) is shown to produce magnetic domains with welldefined DWs (arrowed), suggesting the FeRh is completely FM. The DPC image of Fig. 2c shows the planar FeRh sample positioned on a Lightning ${ }^{\mathrm{TM}} \mathrm{TEM}$ e-chip to be in a FM state at $150^{\circ} \mathrm{C}$ with magnetic domains exhibiting well-resolved DWs, which are then shown in Fig. $2 \mathrm{~d}$ to be driven in an upwards direction by the application of $500 \mu$ s CPs with a current density of $\sim 3.5^{*} 10^{10} \mathrm{~A} / \mathrm{m}^{2}$ (arrowed). Figure 2 has therefore provided evidence for the thermally-induced magnetostructural transition from AF stateq to FM state proceeds via nucleation of small vortex structures, which then grow by combining with newly nucleated vortex states into larger complex magnetic domains. In addition, application of CPs in situ within the TEM allows for visualisation of the current-driven DW motion and calculation of DW velocity.

\section{References:}

[1] L H Lewis et al., J. Phys. D: Appl. Phys. 49 (2016), p. 323002.

[2] X. Marti et al., Nature Mat. 13 (2014), p. 367.

[3] C. Le Graët et al., APL Mater. 3 (2015), p. 041802.

[4] T. P. Almeida et al., J. Phys. Conf. Ser. 903 (2017), p. 012022.

[5] T. P. Almeida et al. Sci. Rep. 7 (2017), p. 17835.
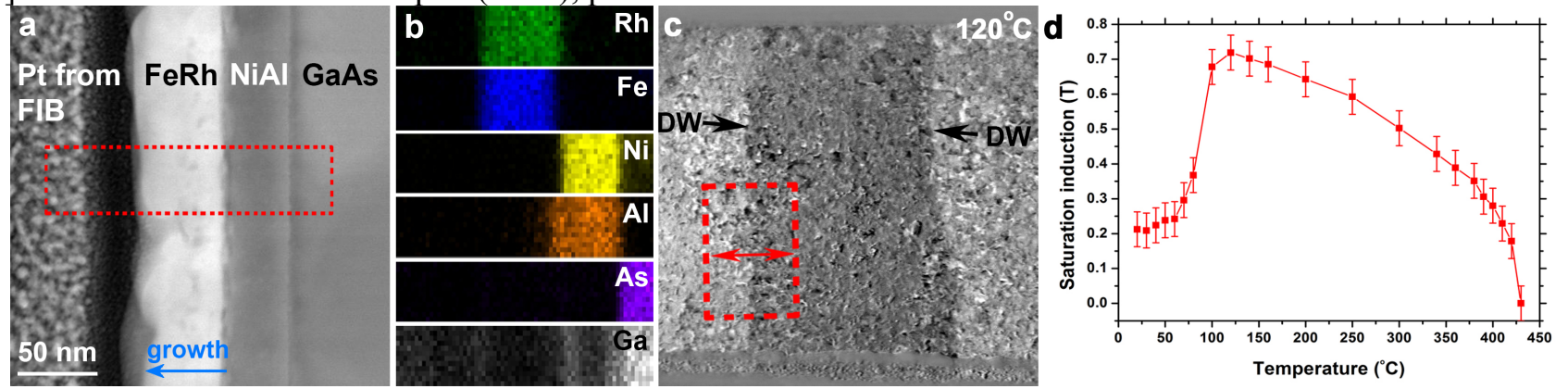

Figure 1. (a) DF STEM image of an FeRh thin film epitaxially grown on a NiAl buffer layer and GaAs substrate ${ }^{5}$. (b) EDX chemical maps acquired from the boxed region in (a), showing their elemental content. (c) DPC image of magnetic domains/DWs in a planar FeRh thin film acquired at $120^{\circ} \mathrm{C}$. (d) Graph of saturation induction as a function of temperature, calculated using direct measurements of electron-beam deflection from the DW in the boxed region (red) defined in (c).
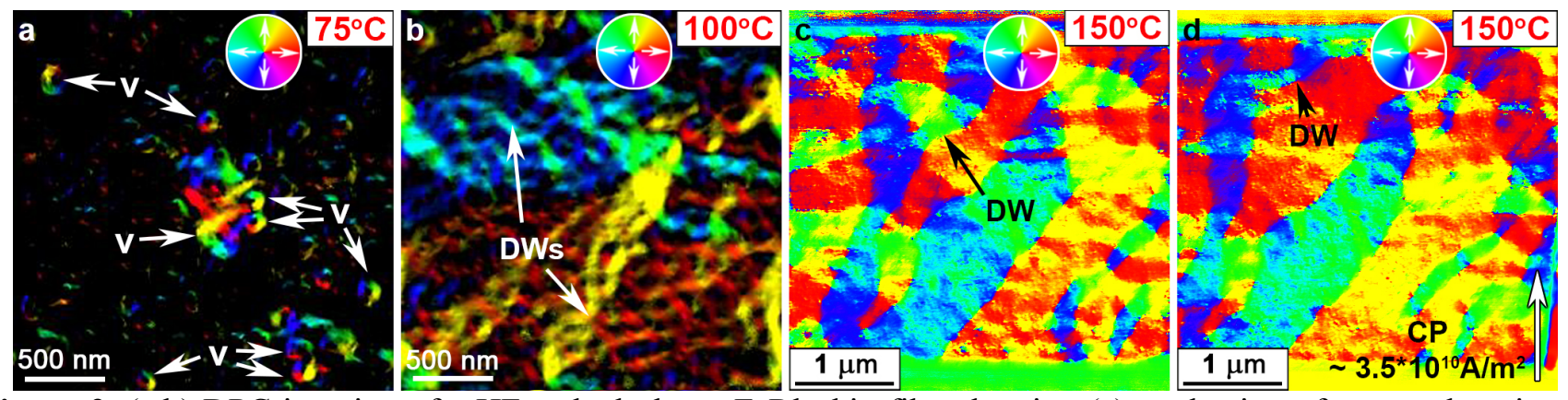

Figure 2. $(a, b)$ DPC imaging of a HF-etched planar FeRh thin film showing (a) nucleation of vortex domains at $80^{\circ} \mathrm{C}$ (denoted by ' $\mathrm{v}$ '); and (b) well-resolved DWs after heating to $120^{\circ} \mathrm{C}$ (arrowed). (c,d) DPC imaging of DWs in a planar FeRh thin film transferred onto a Lightning ${ }^{\mathrm{TM}}$ TEM e-chip heated to (c) $150^{\circ} \mathrm{C}$; and (d) after application of $500 \mu \mathrm{s}$ CPs with a current density of $\sim 3.5^{*} 10^{10} \mathrm{~A} / \mathrm{m}^{2}$ in the arrowed direction. The direction of magnetization in (ad) is depicted in the colour wheels (inset). 